\title{
Sarcoidosis y carcinoma fibrolamelar del hígado
}

\author{
R. PILA PÉREZ, R. PILA PELÁEZ, P. ROSALES TORRES ${ }^{1}$, V. A. HOLGUÍN PRIETO, \\ L. F. ALZATE GIRALDO \\ Servicios de Medicina Interna y ${ }^{1}$ Anatomía Patológica. Hospital Universitario "Manuel \\ Ascunce Domenech". Camagüey. Cuba
}

SARCOIDOSIS AND FIBROLAMELLAR CARCINOMA OF THE LIVER

\begin{abstract}
RESUMEN
Se presenta el caso de una paciente de 20 años de edad con el diagnóstico de sarcoidosis asociada a carcinoma hepático fibrolamelar. Se exponen algunos aspectos clínicos de estas dos enfermedades, así como la conducta diagnóstica y terapéutica seguida. Se resaltan las opiniones de diferentes investigadores sobre la posible relación de la sarcoidosis con las enfermedades neoplásicas, las cuales hasta el momento actual son contradictorias. Se concluye que esta asociación es supremamente rara y debe tenerse en cuenta en el estudio de pacientes jóvenes con sintomatología compatible.
\end{abstract}

PALABRAS CLAVE: Sarcoidosis. Carcinoma hepático fibrolamelar. Asociación.

\section{ABSTRACT}

The case of 20-years female patient with the diagnosis of sarcoidosis associated to fibrolamellar carcinoma of the liver is presented. Clinical features, diagnosis and treatment of these entities are exposed. The criteria of several researchers about the relation between sarcoidosis and neoplasic diseases are stood out. Sarcoidosis associated to fibrolamellar hepatocarcinoma is very uncommon.

KEY WORDS: Sarcoidosis. Fibrolamellar hepatocarcinoma. Association.

Pila Pérez R, Pila Peláez R, Rosales Torres P, Holguín Prieto VA, Alzate Giraldo LF. Sarcoidosis y carcinoma fibrolamelar del hígado. An Med Interna (Madrid) 2007; 24: 431-434.

\section{INTRODUCCIÓN}

La sarcoidosis es una enfermedad de etiología desconocida que se caracteriza por la acumulación en los tejidos afectos de linfocitos CD4+ y monocitos, así como la presencia de granulomas no caseificantes que conducen a la alteración de la arquitectura tisular normal (1). Aunque suele existir anergia cutánea y disminución de los procesos de inmunidad celular en sangre, en los lugares afectados la sarcoidosis se caracteriza por un aumento de la actividad inmunitaria en la que intervienen los linfocitos $\mathrm{T}$ colaboradores (TH1) (1). El HLADRB1 se señala como un factor de riesgo para esta enfermedad (2).

Esta entidad puede afectar cualquier parte del organismo, siendo más frecuente en el pulmón, ganglios, piel, ojos, así como en el hígado y en el bazo. Se ha señalado la asociación de sarcoidosis con enfermedades inmunes (3), neoplasias (4) e infecciones (5).

El hepatocarcinoma fibrolamelar (HF) fue primeramente reportado por Edmonson en 1956, es un tipo especial de hepa- tocarcinoma, dado que la mayoría de los casos ocurren en ausencia de cirrosis del hígado y pueden ser resecables (6); este tumor es muy raro, y lo es más aún en América Latina, donde sólo se han reportado hasta el 2002, 12 casos (7), y en la literatura revisada a nivel mundial, entre 1980 y 1999 se habían informado solamente 165 casos (7).

El objetivo de este trabajo es la presentación de una paciente con sarcoidosis asociada a un HF, asociación que no hemos encontrado reportada en la literatura médica después de un profundo rastreo bibliográfico, y que además constituye el primer caso expuesto en nuestro pais.

\section{CASO APORTADO}

Paciente femenina, negra, de 20 años de edad, estudiante, soltera, sin hábitos tóxicos, ni antecedentes patológicos familiares de interés, refiere que desde los 12 años sufría de bronquitis recurrentes, por lo que fue asistida desde la niñez en consulta de alergología, recibiendo tratamiento con antihistamínicos, esteroides inhalados, y vacunas

Trabajo aceptado: 10 de abril de 2007 
entre otros. En el año 2002, a los 16 años fue ingresada en otro centro hospitalario con el diagnóstico al egreso de neumopatía infecciosa atípica; siendo asistida posteriormente en consultas de alergología y neumología, por crisis de aparente bronquitis alérgica. En el año 2003, aparecen adenopatías supraclaviculares y cervicales derechas, asociadas a un cuadro de bronquitis aguda, manifestaciones clínicas que mejoraron con la terapéutica impuesta. En el año 2005 comienza a presentar dispepsia, dolor en hipocondrio derecho, astenia, anorexia, malestar, pérdida de peso de $7 \mathrm{~kg}$, febrículas vespertinas y adenopatías supraclaviculares y axilares derechas, motivos por los cuales se decide su hospitalización en nuestra institución.

Examen físico. Afectación del estado general, adinámica, hidratada, mucosas normocoloreadas, fiebre de $38^{\circ} \mathrm{C}$.

Aparato cardiorrespiratorio: frecuencia respiratoria: 16 $\mathrm{resp} / \mathrm{min}$. Murmullo vesicular con roncos y sibilantes aislados. No se auscultan soplos. No hay evidencia de arritmias. Tensión arterial: 110/70 mm Hg. Frecuencia cardíaca central: 84 lat/min.

Abdomen: Blando, depresible; a nivel del lóbulo derecho del hígado se palpa una masa de $10 \mathrm{~cm}$ aproximadamente, que afecta casi la totalidad de dicho lóbulo, algo dolorosa, de superficie irregular, bien delimitada. No se constatan otras tumoraciones, ascitis, ni signos de insuficiencia hepática o hipertensión portal.

En la región supraclavicular derecha se hallan 3 a 4 adenopatías de más o menos $3 \mathrm{~cm}$, no dolorosas, adheridas a planos profundos, de consistencia algo dura y temperatura normal. En la región axilar ipsilateral se palpan 2 adenopatías con características similares.

Resto del examen físico: normal, incluyendo examen rectal y vaginal. Fondo de ojo: normal.

Estudio analítico. Hemoglobina: $118 \mathrm{~g} / \mathrm{l}$, hematócrito: 39\%, leucocitos con fórmula diferencial: dentro de la normalidad; velocidad de sedimentación globular: $101 \mathrm{~mm} / 1^{\mathrm{a}} \mathrm{h}$. Glucemia, creatinina sérica, lipidograma, ácido úrico en suero, iones, y enzimas pancreáticas: normales. Conteo de reticulocitos y de plaquetas, tiempos de protrombina, sangrado y coagulación; tiempo parcial de tromboplastina (kaolín), niveles de fibrinógeno: sin alteraciones. TGP: 210 U/1 (Normal < $31 \mathrm{U} / \mathrm{l})$, TGO: $1250 \mathrm{U} / 1$ (Normal < $31 \mathrm{U} / \mathrm{l})$, GGT: $145 \mathrm{U} / 1$ (Normal < 32 U/1), LDH: $520 \mathrm{U} / 1$ (Normal < $460 \mathrm{U} / 1$ ), fosfatasa alcalina: $240 \mathrm{U} / 1$ (Normal < $117 \mathrm{U} / \mathrm{l}), \alpha$-fetoproteína: Normal. $\mathrm{HB}_{\mathrm{s}} \mathrm{Ag}$ : negativo. Anticuerpos contra el virus de la hepatitis $\mathrm{C}$ : negativos. Proteínas totales: 6,2 g/dl, albúmina: 2,3 g/dl, gammaglobulinas de $2,1 \mathrm{~g} / \mathrm{dl}$. Cuantificación de inmunoglobulinas (Ig): Ig G: $1380 \mathrm{mg} / \mathrm{dl}$ (N: $1250 \mathrm{mg} / \mathrm{dl})$, Ig M: $350 \mathrm{mg} / \mathrm{dl}$ (N: $125 \mathrm{mg} / \mathrm{dl})$ e Ig A: $160 \mathrm{mg} / \mathrm{dl}$ (N: $210 \mathrm{mg} / \mathrm{dl}$ ). VDRL y VIH: negativos. Coprocultivo, hemocultivo y urocultivo: no crecimiento. Pruebas en búsqueda de Brucella: negativas. Prueba de Mantoux con 5-PPD: $2 \mathrm{~mm}$. Fenómeno LE, prueba del látex para factor reumatoideo, anticuerpos antinucleares, niveles de complemento sérico: $\mathrm{C} 3, \mathrm{C} 4, \mathrm{C} 5$ y CH50: todos normales.

Electrocardiograma: taquicardia sinusal. Estudio radiológico del tórax: adenopatías hiliares bilaterales. Tomografía axial computarizada (TAC) de mediastino: adenopatías hiliares bilaterales, sin alteraciones adicionales. Biopsia por aspiración con aguja fina $(B A A F)$ de ganglio: no se observan células neoplásicas, muestra con abundantes linfocitos, se sugiere exéresis y estudio histopatológico; se realiza biopsia de ganglio supraclavicular, cuyo informe resulta compatible con una sarcoidosis (Fig. 1).

Ultrasonografía de abdomen: Hígado aumentado de volumen, de ecogenicidad irregular, con masa ecogénica irregular de 108 x 92 $\mathrm{mm}$, que ocupa casi la totalidad del lóbulo derecho; no se aprecian adenopatías periaórticas ni en hilio hepático; no se observan alteraciones adicionales. TAC de hígado: hígado de densidad irregular con masa de baja densidad, no homogénea, de 110x100 mm, en proyección del lóbulo derecho. Después de la administración de contraste se evidencia realce no homogéneo de dicha tumoración; no se reportan otras alteraciones.

$B A A F$ de hígado guíada por ultrasonido: Se aprecia hipercelularidad con hepatocitos grandes, poligonales, de citoplasma abundante, rosado; algunas células con presencia de bilis. Los hepatocitos se encuentran principalmente dispersos, aunque algunos forman peque-

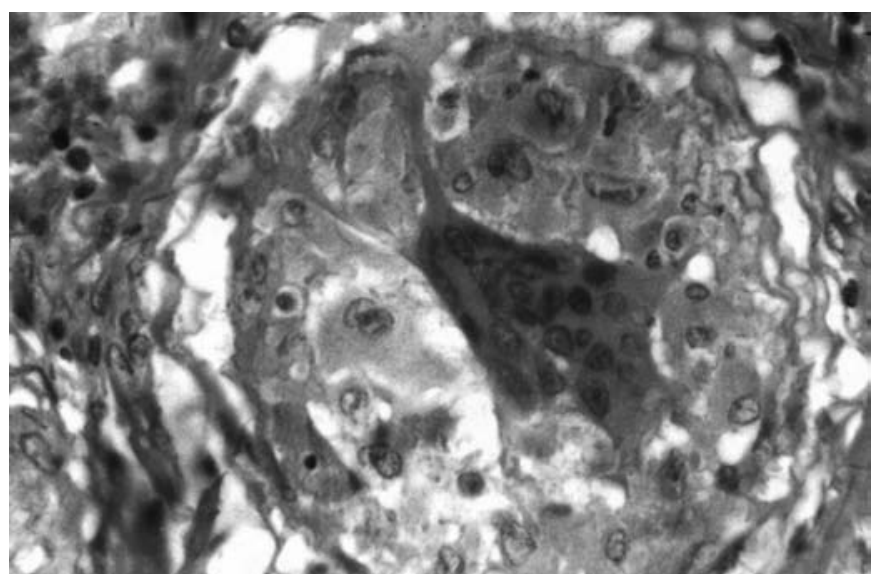

Fig. 1. Imagen histológica de un granuloma sarcoideo. Obsérvese la célula gigante multinucleada (H/E 40X).

ños grupos. Los núcleos son grandes, con nucléolos prominentes, algunas células son binucleadas y con pseudoinclusiones; encontrándose en el extendido pequeños fragmentos de tejido fibroso. El examen se corresponde con un hepatocarcinoma fibrolamelar (HF).

La paciente fue sometida a intervención quirúrgica, encontrándose macroscópicamente un hígado no cirrótico, de aspecto normal, con un tumor único en el lóbulo derecho, de aproximadamente $10 \mathrm{~cm}$, bien delimitado, con cicatriz central, de aspecto lobulado, escirroso, y de color pardo grisáceo. El estudio histopatológico confirma el diagnóstico de un $H F$ (Fig. 2). La paciente recibe el alta hospitalaria y es enviada a nuestra consulta para su seguimiento, encontrándose notablemente recuperada.

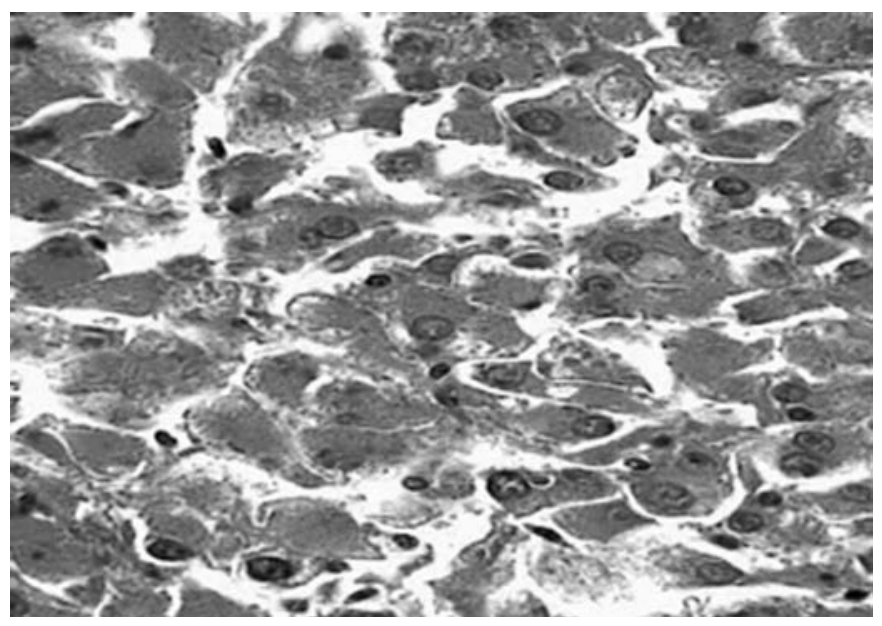

Fig. 2. Imagen a gran aumento donde se observan células malignas con abundante citoplasma de aspecto poligonal (H/E 40x).

\section{DISCUSIÓN}

Por más de 20 años se ha debatido si la sarcoidosis sistémica predispone a las neoplasias malignas, siendo pocos los conocimientos acerca del cáncer en los pacientes portadores de esta entidad. Algunos autores (4) señalan un incremento del riesgo de sufrir cáncer, particularmente del pulmón, estómago, intestino delgado, melanoma, linfoma no-Hodgkin y leucemia; pero se ha señalado que el riesgo es igual o ligeramente superior al observado en otras enfermedades crónicas 
como la diabetes mellitus (8), enfermedades inflamatorias del intestino (9), y artritis reumatoide (10). Las comunicaciones en la bibliografía al respecto son muy discordantes, así, Brincker y Wilbek (11) en 2.544 casos de sarcoidosis encontraron 48 casos con enfermedades neoplásicas respiratorias y linfomatosas, sin que el resto de las neoplasias mostrara un aumento significativo; Battesti y cols. (12), concluyen que no existe un mayor índice de malignidad en las 580 sarcoidosis estudiadas por ellos; Bogaerts y cols. (13), comunican no tener evidencia alguna de que esta patología predisponga a enfermedades malignas. Romer y cols. (14), en un estudio realizado en Dinamarca en 555 pacientes confirman que no hay un aumento de neoplasias malignas en esta enfermedad. Nosotros (15), en 24 años de seguimiento de 110 pacientes, encontramos solamente 3 casos, uno con adenocarcinoma renal, otro con linfoma no-Hodgkin, y otro con cáncer del pulmón. El presente caso fue diagnosticado en el 2005 y no está incluido en la casuística mencionada.

Se ha especulado que el posible incremento de las neoplasias en la sarcoidosis es causado por las deficiencias inmunológicas que son conocidas y que están asociadas a esta entidad (13), o también puede ser debido a la inflamación crónica, la cual actúa como otro importante factor de riesgo (4).

Dentro de los hepatocarcinomas, los HF son extremadamente raros, pues sólo se han reportado aisladamente; en la serie de Kaczynski (16) de 532 casos, solamente se informan 2 HF (0.3\%); en la investigación de Chedid y cols. (17), de 224 pacientes, 4 eran portadores de un HF (1.8\%); mientras que en México, Arista-Nasr y cols. (7), en 121 casos, encontraron $7 \mathrm{HF}(5.8 \%)$. En un estudio realizado por nosotros (18) durante 9 años en esta provincia, se encontraron 78 enfermos de hepatocarcinoma, sin ningún HF reportado.

En referencia al caso en cuestión, se trata de una paciente negra de 20 años de edad, correspondiéndose con todos los autores $(1,2,15,19)$, que señalan que esta enfermedad puede aparecer independientemente del color de la piel, sin embargo, en los Estados Unidos es más frecuente en los negros con una relación de 10-17:1 frente a los blancos. La relación hombre-mujer es aproximadamente de $2: 1$, pero en otros países hay un ligero predominio en mujeres (15), ocurriendo un aumento en frecuencia entre 45 y 65 años de edad (19). Nuestra paciente presentó una pérdida de $7 \mathrm{~kg}$ de peso, fatiga, debilidad, malestar general, síntomas respiratorios de larga fecha y adenopatías. Las adenopatías en la sarcoidosis se reportan con una frecuencia entre 75-90\%, la afectación pulmonar en el $90 \%$, y el compromiso hepático entre el $60-90 \%$, siendo palpable sólo en el $20 \%$ de los casos $(1,15,19)$.

Los hallazgos radiológicos de nuestra paciente se corresponden con el estadio I, es decir, presencia de adenopatías hiliares bilaterales (20). Después de obtener los datos clínicos, imagenológicos y el estudio histopatológico del ganglio que confirmó el diagnóstico de sarcoidosis, se observó que la presencia de la afectación hepática de nuestra paciente no es la señalada por los diferentes autores (21) y que es clasificada en tres distintas formas evolutivas: granulomatosis hepática sarcoidea aislada, granulomatosis hepática sarcoidea con evolución a la fibrosis hepática y granulomatosis hepática sarcoidea masiva con evolución a la cirrosis hepática. Los estudios practicados señalaban más bien un proceso tumoral. Además, debe tenerse en cuenta que la biopsia hepática debe efectuarse de forma sistemática en todo paciente portador de una sarcoidosis, ya que puede existir escasa sintomatología, aún con compromiso de este órgano (21). En nuestra enferma llamó la atención la presencia de hipergammaglobulinemia, la cual es causada por la liberación de linfoquinas de células $\mathrm{T}$ o por estimulación de linfocitos B (19).

El hepatocarcinoma es el tumor primario más frecuente del hígado, reportándose entre el 2-3\% de todos los tumores en los Estados Unidos; dentro de estos tumores, el HF constituye aproximadamente el $10 \%$, y se encuentra en el $35 \%$ de los casos de hepatocarcinomas de pacientes jóvenes sin cirrosis hepática (6).

Los pacientes con HF presentan una supervivencia a los 5 años del 25-30\%, pero puede llegar al 63\%. Si el tumor es resecable, los pacientes pueden alcanzar una supervivencia hasta de 14 años $(6,22)$.

En el HF no existe predominio racial o del sexo, y se trata de pacientes jóvenes con un promedio de 23 años; nuestra enferma tenía solamente 20 años. En la serie de Arista-Nasr y cols. (7), el de mayor edad tenía 26 años y el más joven 17 años. Este tumor se presenta por lo regular con dolor abdominal, masa palpable, hepatomegalia y caquexia $(6,7)$, como ocurrió en nuestro caso. Se señala que a pesar de su gran tamano los pacientes no presentan íctero y la $\alpha$-fetoproteína es negativa, sin embargo, los exámenes de la función hepática pueden estar marcadamente alterados (6), así como fue expuesto en esta paciente.

Cuando se realiza el diagnóstico de esta neoplasia ya está en fases muy avanzadas y el tumor es muy grande, reportándose un diámetro entre 10-20 cm, lo cual pudo ser evidenciado en este caso; sin embargo, no se hallaron adenopatías regionales, las cuales son muy frecuentes (80\%), invasión portal, ni carcinosis peritoneal $(16,17,22)$.

El HF puede ocurrir en cualquier sitio del hígado, nuestra paciente lo presentó en el lóbulo derecho; Arista-Nasr y cols. (7), en su serie señalan que 4 tenían el tumor en el lóbulo derecho y 3 en el lóbulo izquierdo y sus tamaños oscilaron entre 7,4 y $26 \mathrm{~cm}$; el tumor de nuestra paciente tenía una dimensión de aproximadamente $10 \mathrm{~cm}$.

Imagenológicamente se comportan como una masa única o múltiple bien delimitada y con frecuencia muestran una cicatriz central, similar a la encontrada en la hiperplasia nodular focal, por lo que algunos autores (23) señalan que estas entidades pueden estar relacionadas patológicamente. Otro diagnóstico a tener en cuenta es el adenoma de células hepáticas (23).

La biopsia percutánea puede ser definitiva para el diagnóstico pudiéndose realizar bajo control de TAC o ultrasonido, obteniéndose muestras de múltiples sitios $(6,16,17,23)$, tal y como fue realizada en nuestra paciente, y que fue confirmatoria de su padecimiento.

La terapéutica siempre es quirúrgica, por lo regular con la resección primaria del tumor, como ocurrió en este caso. También se puede practicar transplante de hígado con resección en bloque, incluyendo las metástasis ganglionares (6). El tumor puede recurrir y afectar el hígado, pudiendo ser unifocal o multifocal, acompañado o no de adenopatías $(6,7,16,17)$. Los pacientes con lesiones irresecables pueden ser tratados con quimioterapia, ya que la radioterapia en estos casos no es útil $(6,16,22,23)$. 


\section{Bibliografía}

1. Cristal RG. Sarcoidosis. In: Kasper D, Braunwald E, Fauci A, Hauser S, Longo D, Jameson JL, editors. Harrison's Principles of Internal Medicine. 16th ed. New York: McGraw-Hill; 2005. p. 2017-2023.

2. Rossman M, Thompson B, Frederick M, Maliarick M, Ianuzzi M, Rybiki B, et al. HLA-DRB1: A significant risk factor for sarcoidosis in black and white. Am J Hum Genet 2003; 73:720-735.

3. Eznnauver R, West S. Sarcoidosis in autoimmune disease. Semin Arthritis Rheum 1993; 22: 1-9.

4. Askling J, Grunewald J, Eklund A, Hillerdal G, Ekbomb A. Increased risk for cancer following sarcoidosis. Available from: http://ajrccm.atsjournals.org/cgi/content/full/160/5/1668

5. Pila Pérez R, Pila Peláez R, Paulino Basulto M, Del Sol Sosa J. Sarcoidosis y brucelosis: Una extraña e infrecuente asociación. Gac Med Mex 2003; 139: 160-4.

6. Haddad JL, Coombs BD, Schmiedl UP, Krasny RM, Karani J, editors. Hepatocellular carcinoma, Fibrolamellar [monograph on the Internet]. New York: www.emedicine.com; 2007 [Last updated 2007 Apr 27]. Available from: www.emedicine.com/radio/topic333.htm

7. Arista-Nasr J, Gutiérrez Villalobos L, Nuncio J, Maldonado H, Borstein Quevedo L. Fibrolamellar hepatocellular carcinoma in mexican patients. Pathol Oncol Res 2002; 8: 133-7.

8. Adami H, McLaughlin A, Ekbomb C, Berne D, Silverman D, Hacker $\mathrm{D}$, et al. Cancer risk in patients with diabetes mellitus. Cancer Cases Control 1991; 2: 307-14.

9. Ekbomb A, Helmick M, Zack C, Adami H. Extracolonic malignancies in inflammatory bowel disease. Cancer 1991; 67: 2015-19.

10. Gridley G, McLaughlin A, Ekbomb A, Klareskog L, Adami H, Hacker $\mathrm{D}$, et al: Incidence of cancer among patients with rheumatoid arthritis. J Natl Cancer Inst 1993; 85: 307-11.

11. Brincker $\mathrm{H}$, Wilbek $\mathrm{E}$. The incidence of malignant tumors in patients with respiratory sarcoidosis. Br J Cancer 1974; 29: 247-51.
12. Battesti J, Turiaf J, Hincky J, Duornovo P. Reactions sarcoidiennes loco regionales. Sarcoidose et tumeurs malignes. Nov Presse Med 1977; 6: 1213-15.

13. Bogaerts M, Vander Straeten J, Tasson F, Pawls R. Sarcoidosis or malignancy: A diagnostic dilemma. Eur J Respir Dis 1983; 64: 541-50.

14. Romer F, Hommelgaad P, Schou G. Sarcoidosis and cancer revisited A long term follow-up study of 555 Danish sarcoidosis patients. Eur Respir J 1998; 12: 906-12.

15. Pila Pérez R, Pila Peláez R, Pila Peláez M. Sarcoidosis: Estudios y resultados en 110 pacientes. Archivos de la Facultad de Medicina de Zaragoza 2004; 44: 106-112.

16. Kaczynski J, Gustavsson B, Hannson G. Fibrolamellar Hepatic carcinoma in an area with low incidente of primary liver cancer: A retros pective study. Eur J Surg 1996; 162: 367-71.

17. Chedid A, Ryan L, Dayal Y. Morphology and other prognostic factors of hepatocellular carcinoma. Arch Pathol Lab Med 1999; 123: 524-8.

18. Segura Herrera R, Pila Pérez R, Pila Peláez R. Carcinoma hepático primario en Camagüey (Cuba). Enfermedades Emergentes 2004; 6: 17 23.

19. Amorosa JK, Coombs BD, Webb WR, Krasny RM, White CS, editors Sarcoidosis, Thoracic [monograph on the Internet]. New York: www.emedicine.com; 2007 [Last updated 2005 March 1]. Available from: www.emedicine.com/radio/topic618.htm

20. Gotway M, Tchao N, Leung J. Sarcoidosis presenting as an enlarging solitary pulmonary nodule. J Thorac Imaging 2001; 16: 117-22.

21. González Escalada J, Teigelle Cea J, Muñoz Castillo M, Franco Manera R. Sarcoidosis hepática. Rev Clin Esp 1980; 159: 229-35.

22. McLamey J, Rucker P, Bender G. Fibrolamellar carcinoma of the liver: Radiologic-pathologic correlation. Radiographics 1999; 19: 453-71.

23. Ptiman M. Fine needle aspiration of the liver. Principal diagnostic challenges. Clin In Lab Med 1998; 18: 483-506. 